\title{
Caféiculture Et Accès Aux Soins De Santé Au Burundi : Analyse De La Dynamique Des Mutuelles De Santé Des Caféiculteurs De La Province Kayanza Au Nord Du Burundi
}

\begin{abstract}
Ndabarushimana Alexis
Docteur en sociologie du développement rural

Directeur Adjoint Chargé de la Formation Diplômante et de la Recherche à l'Ecole Nationale d'Administration/Bujumbura et Enseignant-chercheur de sociologie du développement rural à l'Université Espoir d'Afrique,

Département de Service Social et

Développement Communautaire et de management public à 1'ENA
\end{abstract}

Doi: 10.19044/esj.2018.v14n3p427 URL:http://dx.doi.org/10.19044/esj.2018.v14n3p427

\begin{abstract}
This article highlights the dynamics of health mutuals of coffee farmers in Kayanza province. It shows the factors related to difficulties in accessing health care for coffee growers in Kayanza province. These factors are fundamentally linked to the socio-economic environment that structures the living conditions of coffee growers in a world where the Burundian government has opted a "laissez-faire" and "laissez-aller" policy in the coffee sector. In order to do this, we opted for qualitative approaches with the use of the interview guide and quantitative with the use of a questionnaire administered directly to the coffee growers of Kayanza province. In this situation of self-determination of coffee growers, this article highlights the constraints faced by mutual health organizations in their mission to be able to access health care quality of coffee farmers after having shown the role played by mutuals and FEMUSCABU in promoting the solidarity of coffee farmers in Kayanza province. This article also highlights how mutualists behave with their social vulnerability.
\end{abstract}

Keywords: Quality Health Care, Mutual Health

\section{Résumé}

Cet article met en lumière la dynamique des mutuelles de santé des caféiculteurs de la province Kayanza. Il montre les facteurs liés aux difficultés d'accès aux soins de santé chez les caféiculteurs de la province Kayanza. Ces facteurs sont fondamentalement liés à l'environnement socio-économique qui 
structure les conditions de vie des caféiculteurs dans un monde où l'Etat burundais a opté pour la politique du "laisser-faire,', "laisser-aller', dans le secteur café. Pour y arriver, nous avons opté pour des approches qualitative avec l'usage du guide d'interview et quantitative avec l'usage d'un questionnaire administré de façon directe aux caféiculteurs mutualistes de la province Kayanza. Face à cette situation d'autodétermination des caféiculteurs, cet article met en évidence les contraintes auxquelles font face les mutuelles de santé dans leur mission de pouvoir l'accès aux soins de santé des qualité des caféiculteurs mutualistes après avoir montré le rôle que jouent les mutuelles de santé et la FEMUSCABU dans cette promotion de la solidarité des caféiculteurs mutualistes de la province Kayanza. Cet article met aussi en évidence la manière dont les mutualistes se comportent face à leur vulnérabilité sociale.

Mots-clés : Soins de santé de qualité, Mutuelle de santé

\section{Introduction}

L'Afrique rurale et plus particulièrement le Burundi se trouve dans un processus interminable de déstructuration, de restructuration et d'adaptation aux nouvelles exigences du développement communautaire. La conquête donc du bien être des communautés a toujours constitué une préoccupation majeure des gouvernants burundais.

Se faisant, personne ne peut nier, comme Mondjanagni (1984) le disait "le développement implique nécessairement des transformations qualitatives et des modifications des structures sociales et économiques"' (p. 24).

Situé aux confins de l'Afrique centrale et de l'Afrique de l'Est, le Burundi est un pays historiquement agricole. Son économie repose essentiellement sur une agriculture de subsistance, caractérisée par une très forte population agricole (plus de $90 \%$ de la population totale), un émiettement des exploitations (moyenne inférieure à 0,5 ha) et une très faible productivité.

A côté des cultures vivrières qui assurent la subsistance, la population rurale burundaise développe aussi les cultures de rente, surtout le café et le thé qui génèrent, elles seules, $90 \%$ des recettes d'exportation et contribuent à 7,5 $\%$ au Produit Intérieur Brut. (Ministère de l'Agriculture et de l'Elevage, 2008). Le café à lui seul contribuait à hauteur de 5\% entre 2008 et 2009 selon le rapport final du projet $\mathrm{CFC} / \mathrm{OIC} / 30$ qui visait à “'encourager l'intensification de la production du café et des cultures vivrières par l'utilisation de la fumure organique dans les zones couvertes par le projet au Burundi', . De 2001 à 2010, la part du café aux recettes d'exportation représentait une moyenne de $63 \%$. L'utilité de cette culture dite de rente a fait qu'elle un enjeu de pouvoir et de spéculation internationale jusqu'en 1991. 
L'encadrement dans le secteur café se faisait jusqu'en 1991 d'une manière policée et policière, malgré les tentatives pour faire adhérer le paysan à travers la méthode de vulgarisation dite participative (Cochet, 2001). C'est une année plus tard que le gouvernement burundais s'est engagé dans une privatisation "limitée" du sous-secteur café, à travers son retrait de toute gestion directe du secteur café.

Entre 1992 et 2005, ce sont les Directions Provinciales de l'Agriculture et de l'Elevage (DPAE) qui s'occupaient de l'encadrement technique caféicole avec moins de pression sur les caféiculteurs.

C'est donc après la promulgation du "décret $n^{\circ}$ 100/012 du 14 janvier 2005 portant reforme de la filière café' 'que l'encadrement technique des caféiculteurs ait été confié aux privés qui s'organisent depuis lors au sein des fédérations des caféiculteurs. Selon ce décret, La liberté d'établissement et d'exercice dans tous les maillons de la chaine de production, de commercialisation, de transformation, de l'exportation et de financement dans le secteur café est reconnue à tout opérateur économique qui le souhaite. C'est dans ce contexte que la Fédération des associations des caféiculteurs « BONAKURE » de la province Kayanza a été créée le 30/10/2003 et agréée le 23/11/2004. Elle est composée de 27 unions réunissant actuellement 677 associations.

Toutes ces structures rassemblent actuellement 21347 caféiculteurs. La Fédération a un champ d'action qui s'étend sur 3 provinces à savoir la province de Kayanza, une partie de la province Ngozi en commune Busiga et une partie de la province Muramvya en commune Bukeye. A partir de 2005, le caféiculteur recouvre sa liberté dans toutes les activités liées à la production du café y compris le secteur de la santé.

Depuis les années 60, le système de santé en Afrique commença à être difficilement maîtrisé et la part réelle des dépenses publiques dans le secteur de la santé diminua progressivement dans de nombreux pays africains au cours des années 70. comme Ndabarushimana (1999) le disait, même les Politiques d'Ajustement Structurel (PAS) initiées au début des années 80 dans les pays en développement et à partir de 1986 au Burundi, n’ont pas réussi à améliorer les systèmes de santé. C'est l'année 1991 qui marque pratiquement la fin du PAS au Burundi et le début du processus de privatisation de 1992 à 2005 qui s'est concrétisé en 2005 par la signature du décret $n^{\circ} 100 / 012$ du 14 janvier 2005 portant réforme de la filière café.

Sur la période de mise en œuvre du CSLP II, le gouvernement burundais avait ciblé l'adhésion à un système d'assurance maladie d'au moins $50 \%$ de la population du secteur informel et $40 \%$ du secteur rural et la vulgarisation d'autres paliers de la protection sociale tels que les pensions et les synergies entre croissance économique et protection sociale. Et le sentiment des caféiculteurs d'être abandonnés par le gouvernement les a 
poussés à créer leurs mutuelles de santé dans l'espoir d'avoir un accès aux soins de santé.

Toutefois, au cours de la période de mise en œuvre du CSLP I, le gouvernement burundais avait déjà entrepris trois grandes réformes, notamment dans lesdomaines de la gratuité des soins pour les enfants de moins de cinq ans et les femmes enceintes, la décentralisation et les modes de financement avec l'introduction du Financement Basé sur les Performances (FBP), couplé à la gratuité (Gouvernement burundais, 2012) .

Dans le cadre de la mise en œuvre de la Politique Nationale de Développement Sanitaire, PNDS 2011-2015, le gouvernement burundais a poursuivi des réformes de décentralisation afin d'aboutir à des districts sanitaires autonomes, financièrement viables et visant à répondre aux besoins de santé régionaux de la population.

Ces efforts du gouvernement burundais ont été appuyés par les caféiculteurs burundais qui, réunies au sein des associations des caféiculteurs, ont créé 27 mutuelles de santé depuis 2008. La Fédération des Mutuelles de Santé des caféiculteurs du Burundi (FEMUSCABU) apparait alors comme une faitière des mutuelles de santé communautaire. Créée le 14 juin 2011, elle fût agréée par le Ministère Burundais de la Fonction Publique, du Travail et de la Sécurité Sociale le 8 août 2014 par l'ordonnance Ministérielle N570/98.

La présente étude concerne les 5 mutuelles de santé des caféiculteurs réunissant les membres des associations des caféiculteurs membres de la fédération bonakure de la province kayanza. il s'agit de «Tugiramagara meza» de la commune Kabarore, «Tugarukirubuzima» de la commune Mparamirundi, «Kingirubuzima» de la commune Kayanza, «Kira» de la commune Gatara et «Magaranikindi » de la commune Matongo. Elle se fonde sur notre souci d'élargir nos connaissances sur la capacité de survie des caféiculteurs de la province Kayanza après la libéralisation du secteur café en 2005.

A partir de 2011 toutes les mutuelles de santé s’organisent au sein de la Fédération des Mutuelles de Santé des Caféiculteurs du Burundi (FEMUSCABU). La raison d'être de celle-ci étant de promouvoir le renforcement mutuel et la solidarité, faire le plaidoyer en matière de protection sociale et de développement du mouvement mutualiste au Burundi.

Mais aujourd'hui, le constat est que malgré la volonté du gouvernement burundais à renforcer le socle de la protection sociale sur la période de mise en œuvre du CSLP II et les efforts de la FEMUSCABU, les caféiculteurs rencontrent des difficultés à accéder aux soins de santé de qualité.

Dans ce contexte d'auto prise en charge en matière d'accès aux soins de santé avec l'objectif de permettre aux caféiculteurs d'accéder aux soins de santé de qualité, la question de prise en charge se pose dans le sens de la 
dynamique des mutuelles de santé des caféiculteurs. Aussi, l'accès aux médicaments selon le cas demeure-t-il un obstacle majeur.

Quelle est alors la dynamique des mutuelles de santé des caféiculteurs de la province Kayanza ?

En d'autres termes, quelles sont les facteurs liés aux difficultés d'accès aux soins de santé de qualité chez les caféiculteurs mutualistes de la province Kayanza ?

Quel est le rôle des mutuelles de santé des caféiculteurs dans la promotion de l'accès aux soins de santé en province Kayanza ?

Quelles sont les contraintes auxquelles font face les mutuelles de santé des caféiculteurs de la province Kayanza dans leur volonté de permettre à tous les mutualistes d'accéder aux soins de santé de qualité ?

Mieux encore, comment les mutualistes se représentent-ils la dynamique de leur mutuelle de santé et comment se comportent-ils face à leur vulnérabilité sociale?

L'objectif principal de cet article est d'analyser la dynamique des mutuelles de santé des caféiculteurs de la province Kayanza au nord du Burundi. En d'autres termes, cette étude permet de saisir les facteurs explicatifs du non accès aux soins de santé de qualité en province Kayanza, de montrer le rôle des mutuelles de santé dans la promotion de l'accès aux soins de santé, d'élucider les contraintes auxquelles font face les mutuelles de santé dans leur mission de promouvoir l'accès aux soins de santé de qualité des mutualistes et d'appréhender les comportements des mutualistes face à leur vulnérabilité sociale. A notre connaissance, peu d'études empiriques ont été réalisées à ce jour sur ce sujet au Burundi en général et aucune sur ces caféiculteurs de la province Kayanza en particulier. Ceci constitue donc une contribution empirique indéniable. De ce fait, nous présentons d'abord l'introduction suivie de la méthodologie et de la présentation des résultats que nous discutons par la suite avant de conclure en fin.

\section{Concepts et méthodologie}

Se sentant en quelques sorte abandonnés par le pouvoir public, les caféiculteurs burundais commencent à mettre en place leurs propres "mutuelles de santé" dans l'espoir d'avoir accès aux "soins de santé de qualité,', deux concepts centraux de notre analyse et que nous définissons ainsi : permettant de comprendre la réaction des caféiculteurs qui avaient le sentiment d'être abandonnés par le pouvoir public, les caféiculteurs burundais ont commencé par s'organiser au sein des associations des caféiculteurs. Ces associations se sont par la suite organisées en unions et en fédération au niveau provincial.

Pour mieux plaider la cause du caféiculteurs, les fédérations se sont réunies au sein de la Confédération Nationale des Caféiculteurs du Burundi 
(CNAC). Avec le même schéma, les mêmes caféiculteurs ont créé les mutuelles de santé qui se sont ensuite réunies au sein de la Fédération des Mutuelles de Santé des Caféiculteurs du Burundi (FEMUSCABU).

Les mutuelles de santé sont considérées dans la logique des caféiculteurs comme une solution à leurs problèmes d'accès aux soins de santé de qualité, la qualité ici étant à comprendre comme étant cette possibilité offertes aux caféiculteurs au niveau de six aspects : être bien accueilli par les structures de soins, être soigné par un personnel soignant compétent, accéder aux soins à des coûts abordables, pouvoir faire des examens médicaux avant la prescription des médicaments, se faire soigner dans des structures de soins propres et avoir des médicaments disponibles dans les structures de soins. Ces six aspects sur lesquels nous nous basons pour définir le concept "soins de santé de qualité" sont des variables de référence que Kaneza (2013) a utilisé pour évaluer la qualité des soins dont bénéficient les caféiculteurs mutualistes de la part des structures de santé qui ont signé la convention avec les mutuelles de santé. Ainsi, nous dirons que le mutualiste a accès aux soins de santé de qualité lorsque le processus suivi dans les structures de santé répond à tous ces critères.

Une Mutuelle de Santé est un groupe de personnes s'organisant pour faire face, au moyen de leurs seules cotisations, aux conséquences d'un risque social les menaçant. Elle émane d'une volonté des personnes de se regrouper entre elles. Les mutuelles de santé se fondent généralement sur les valeurs de "'non-lucrativité", "solidarité", "volontariat", "démocratie", "indépendance", "'transparence", etc.

Dans le cadre de cet article, nous retiendrons la définition que donne le SAILD (2010) telle que repris par Sobngwi (2011) selon laquelle «une mutuelle de santé est une association démocratique, autonome à but non lucratif, dirigée par ses membres et qui fonctionne sur la base de la solidarité, et essentiellement au moyen des cotisations des membres pour la mutualisation du risque maladie, ayant comme objectif d'améliorer l'accès de ses membres et leurs familles à des soins de santé » (p. 29).

Les mutuelles de santé des caféiculteurs de la province Kayanza dont il est question dans cette étude, sont des associations sans but lucratif, autonomes et qui fonctionnent sur la base de la solidarité, et essentiellement au moyen des cotisations des membres pour la promotion de l'accès aux soins de santé de qualité de ses membres, ayant comme objectif de promouvoir la solidarité et l'accès aux soins de santé de qualité.

La zone d'investigation est constituée de 5 communes dont 4 de la province Kayanza et une zone de la commune Busiga de la province Ngozi. Il s'agit des communes Matongo, Gatara, Kayanza et Kabarore en province Kayanza auxquelles nous ajoutons la zone Mparamirundi de la commune Busiga en province Ngozi. Les 5 communes constituent une localité spécifique 
de production de café et comme cette subdivision avait été établie par la Fédération des Mutuelles de Santé des Caféiculteurs du Burundi FEMUSCABU, nous avons opté pour l'élargissement de notre champ d'étude en gardant la localité de Mparamirundi.

La zone d'investigation telle que définie, bénéficie de l'intervention de l'Union Grand Nord des mutuelles de santé en terme d'encadrement des caféiculteurs mutualistes et offre des éléments factuels d'analyse des logiques socioéconomiques permettant de saisir la dynamique des mutuelles de santé des caféiculteurs.

L'approche adoptée dans cette étude est à la fois qualitative avec les entretiens individuels et quantitative avec un questionnaire administré de façon directe aux mutualistes en tant qu'acteurs principaux et bénéficiaires des actions de leur mutuelles de santé. Nous avons opté pour des entretiens individuels avec les responsables des mutuelles de santé pour éviter les éventuelles influences pouvant avoir lieu au moment de l'interview.

Les données de la base des données de l'Appui au Développement Intégral et la Solidarité sur les Collines (ADISCO) en tant que principal encadreur technique de tous les mutuelles de santé des caféiculteurs du Burundi, montre qu'au cours de l'Exercice 2016-2017, les caféiculteurs mutualistes de notre champ d'étude étaient au nombre de 717 dont 519 hommes et 198 femmes.

Selon Mucchielli (1991), on appelle population d'enquête, «l'ensemble du groupe humain concerné par les objectifs de l'enquête.C'est dans cet univers que sera découpé l'échantillon » (p. 16). C'est aussi, selon N'da (2002), "un ensemble d'unités élémentaires (une personne, un groupe, une ville, un pays) qui partagent des caractéristiques communes précises selon des critères définis', (p. 65).

Compte tenu des spécificités de notre étude, nous souscrivons à l'idée de Ghiglione, \& Matalon (1978) selon laquelle : “il est inutile d'interroger toute la population de l'étude',(p. 29).

Compte tenu des effectifs élevés des caféiculteurs mutualistes de notre champ d'étude, le questionnaire a été administré au près de soixante douze mutualistes représentant $10 \%$ de toute la population d'enquête. Pour déterminer l'échantillon, nous nous sommes basés sur la remarque de Javeau qui stipule que les théories statistiques ne sont pas à appliquer toujours à la lettre mais qu'aucun échantillon ne devrait pas compter moins de trente individus (Javeau, 1990).

Ainsi, dans une population de 717 individus et compte tenu des contraintes temporelles et financières, notre choix a porté sur les acteurs de sexe différents. Ce choix théorique nous a permis d'identifier, à travers la technique d'échantillonnage stratifié, 72 individus dont 52 hommes et 20 femmes. L'analyse des données factuelles recueillies a été faite à l'aide de la 
méthode dialectique qui a contribué à déterminer les facteurs à l'œuvre dans l'accès aux soins de santé de qualité chez les caféiculteurs membres des mutuelles de santé en province Kayanza.

Pour choisir les 72 individus, nous avons utilisé la "technique d'échantillonnage stratifié', Dans chaque zone d'enquête, la stratégie utilisée pour choisir un échantillon représentatif a été la constitution des strates constituées d'éléments ayant les mêmes caractéristiques, c'est-à-dire celle constituées d'hommes d'un côté et de femmes de l'autre. C'est donc à l'intérieur de chaque strate que nous avons tiré au hasard le dixième des éléments constitutifs de chaque strate.

En tant que technique non-probabiliste, l'échantillonnage stratifié a été utilisé pour choisir délibérément les 52 individus de sexe masculin dans 519 et 20 individus de sexe féminin dans 198 en considérant que toutes les femmes caféicultrice membres d'une même mutuelle de santé sont au même niveau d'information par rapport à la dynamique de leur mutuelle de santé. Cette même considération nous a guidés quand il s'agissait de choisir 52 individus de sexe masculin à l'intérieur d'une strate de 519 caféiculteurs membre d'une même mutuelle de santé.

Tableau1 : Répartition des mutualistes par zone d'études en 2017 et échantillon choisi

\begin{tabular}{|c|c|c|c|c|c|c|c|c|c|c|c|c|}
\hline \multirow[t]{2}{*}{ Enquêtés } & \multicolumn{10}{|c|}{ Population et échantillon par zone d'enquête } & \multicolumn{2}{|c|}{ Total } \\
\hline & Gat & $\mathrm{E}$ & $\mathrm{Kab}$ & $\mathrm{E}$ & Kay & $\mathrm{E}$ & Mat & $\mathrm{E}$ & $\mathrm{Mpa}$ & $\mathrm{E}$ & $\mathrm{P}$ & $\mathrm{E}$ \\
\hline Homme & 136 & 14 & 59 & 6 & 108 & 10 & 159 & 16 & 57 & 6 & 519 & 52 \\
\hline Femme & 64 & 6 & 24 & 3 & 53 & 5 & 42 & 4 & 15 & 2 & 198 & 20 \\
\hline Total & 200 & 20 & 83 & 9 & 161 & 15 & 201 & 20 & 82 & 8 & 717 & 72 \\
\hline
\end{tabular}

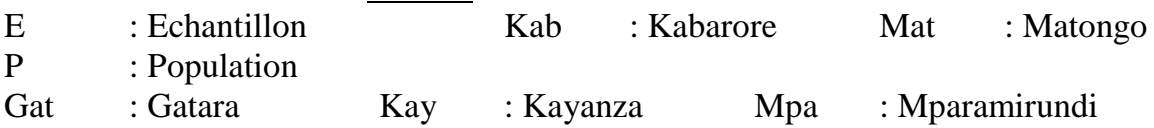

\section{Résultats}

\section{Caractéristiques socio-démographiques des enquêtés}

L'analyse des caractéristiques socio-démographiques des enquêtés a été effectuée à partir de trois variables indépendantes que sont la situation matrimoniale, le nombre d'enfants à charge et le niveau d'instruction.

Tableau 2 : Identification des enquêtés selon la situation matrimoniale et le nombre

\begin{tabular}{|c|c|c|c|c|c|c|c|c|c|}
\hline \multicolumn{10}{|c|}{ d'enfants à charge } \\
\hline \multirow[b]{2}{*}{ N.E.C. } & ba & bin & & & iviance & uf & cé & & \\
\hline & & & $\begin{array}{c}\text { Civ } \\
\text { il }\end{array}$ & $\begin{array}{l}\text { Religie } \\
\text { ux }\end{array}$ & $\begin{array}{c}\text { Civil et } \\
\text { Religieux }\end{array}$ & & & $\begin{array}{l}\text { V } \\
\text { A }\end{array}$ & $\begin{array}{l}\text { VR } \\
(\%)\end{array}$ \\
\hline $\begin{array}{c}\text { Sans } \\
\text { enfant }\end{array}$ & 3 & 0 & 0 & 0 & 0 & 0 & 0 & 3 & $\begin{array}{c}4,1 \\
7\end{array}$ \\
\hline 1 à 3 & 0 & 0 & 0 & 0 & 11 & 1 & 0 & 12 & $\begin{array}{l}16, \\
67\end{array}$ \\
\hline
\end{tabular}




\begin{tabular}{|c|c|c|c|c|c|c|c|c|c|c|}
\hline \multicolumn{2}{|c|}{4 à 6} & 1 & 0 & 2 & 0 & 36 & 1 & 1 & 41 & $\begin{array}{c}56, \\
94\end{array}$ \\
\hline \multicolumn{2}{|c|}{7 et +} & 0 & 0 & 1 & 0 & 15 & 0 & 0 & 16 & 22, \\
22
\end{tabular}

S.M : Situation Matrimoniale

V.A. : Valeur Absolue

Source: Données de l'enquête, août 2017.
N.E.C. : Nombre d'Enfant à Charge

V.R. : Valeur Relative

Les données portées dans le tableau ci-dessus révèlent que la majorité d'enquêtés $(56,94 \%)$ ont entre 4 et 6 enfants; ceux-ci représente environ trois cinquième $(3 / 5)$ de nos enquêtés. Ce résultat s'explique par le fait que le milieu rural burundais reste encore marqué par les valeurs traditionnelles qui structurent la conception de l'enfant et surtout de son importance dans l'environnement socio-économique (richesse du lignage). Pour les enquêtés en majorité, imprégnés des réalités sociales, les enfants constituent un facteur d'intégration sociale.

Les chiffres du tableau ci-dessus nous font remarquer une forte proportion de mutualistes ayant fait le mariage civil et religieux $(86,11 \%)$. On note que parmi tous les enquêtés il n'ya aucun concubin ni divorcé et aucun n'a fait un mariage religieux uniquement.

En effet, ces proportions expliquent les faits sociaux suivants :

d'abord, le mariage légal constitue un facteur d'intégration sociale dans la mesure où un homme marié est celui-là que la communauté respecte par rapport au célibataire. Aussi, selon la loi burundaise en vigueur, les prêtres et les pasteurs ne sont pas autorisés à bénir un mariage sur le plan religieux avant que le mariage civil n'est lieu.

Ensuite, l'existence de seulement trois mutualistes ayant seulement fait un mariage civil s'explique par le fait que le mariage religieux exige souvent des dépenses que l'enquêté ne trouve pas facilement. C'est cette situation qui justifie le décalage entre le mariage civil et le mariage religieux surtout que le mariage civil sans mariage religieux n'est pas condamnable par la loi en vigueur au Burundi. Ainsi, la constitution du Burundi promulguée le 18 mars 2005, précise, en son article premier que "Le Burundi est une République indépendante, souveraine, laïque, démocratique, unitaire et respectant sa diversité ethnique et religieuse'.

Enfin, les veuves restent propriétaires des plantations lorsque le décès de leurs conjoints survient après le mariage civil ou mariage civil et religieux. 
Après l'analyse de la situation des enquêtés selon la situation matrimoniale et le nombre d'enfants à charge, il convient d'analyser leur niveau d'instruction.

Tableau 3 : Identification des enquêtés selon le niveau d'instruction (N.I.) et le sexe

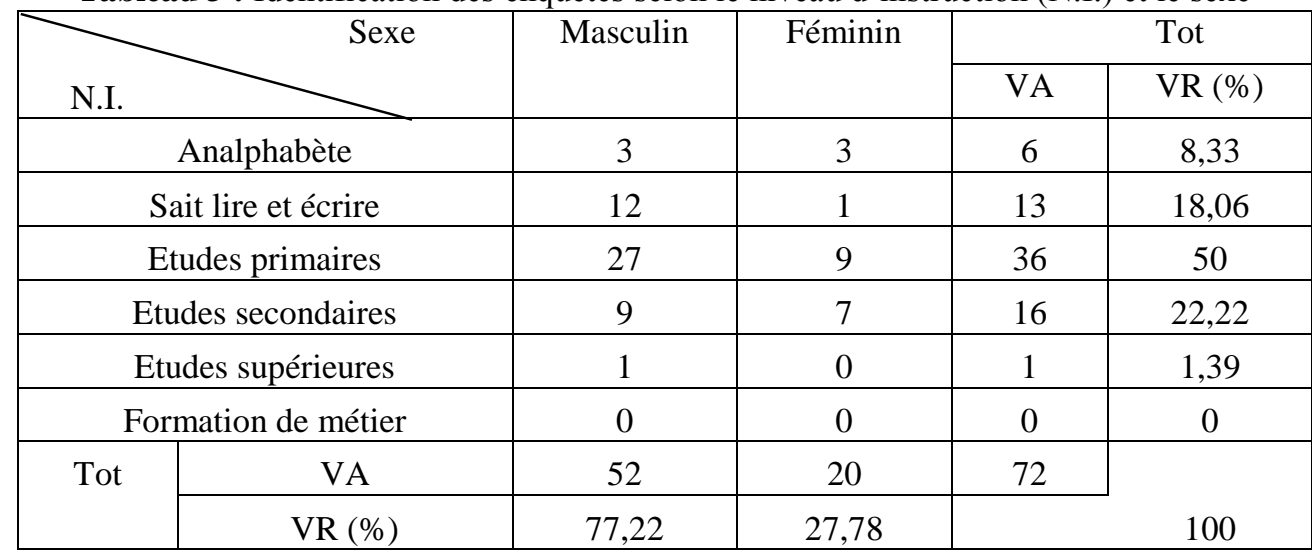

Source: Données de l'enquête, août 2017

Il ressort ici que l'adhésion à une mutuelle de santé est liée au niveau d'instruction des caféiculteurs. En effet, comme l'indique le tableau ci-haut, les analphabètes ne représentent que $8,33 \%$. Environ $4 / 5$ de caféiculteurs qui savent lire et écrire et qui ont un niveau d'étude primaire ont adhéré aux mutuelles de santé. Par contre, c'est seulement 23,61\% qui ont un niveau secondaire et supérieur. Ce tableau renseigne également qu'aucun enquêté n'a suivi une formation dans un métier quelconque.

On en conclut que, plus on sort de la catégorie des analphabètes, plus on a tendance à adhérer à une mutuelle de santé. Cela s'explique par les exigences qui accompagnent l'adhésion à une mutuelle de santé et qui conduisent le paysan à mobiliser ses propres moyens pour s'acquitter de ses cotisations.

En outre, adhérer à une mutuelle de santé des caféiculteurs apparaît aussi aujourd'hui comme un facteur d'intégration sociale dans ce sens que être mutualiste permet de faire comprendre à l'entourage qu'on a des revenus qui lui permettent de s'acquitter de ses cotisations annuelles exigées par la mutuelle elle-même. Et si les hommes représentent des proportions élevées au sein des mutuelles de santé des caféiculteurs c'est parce que le café en tant que culture de rente et stratégique dans un système social patriarcale constitue une source de revenus important au sein des familles des caféiculteurs.

\section{Conditions de vie des caféiculteurs mutualistes}

L'analyse des conditions de vie des caféiculteurs mutualistes a été effectuée à partir de cinq variables indépendantes que sont le revenu, l'accès à 
l'eau potable, type d'habitat, électrification de l'habitat et la scolarisation des enfants.

Tableau 4 : Revenus des mutualistes exprimés en milliers de franc burundais

\begin{tabular}{|c|c|c|}
\hline \multirow[t]{2}{*}{ Revenus } & \multicolumn{2}{|c|}{ Enquêtés } \\
\hline & Valeur Absolue & Valeur Relative $(\%)$ \\
\hline Moins de 100 & 2 & 2,78 \\
\hline Entre 100 et 300 & 12 & 16,66 \\
\hline Entre 300 et 600 & 19 & 26,39 \\
\hline Entre 600 et 900 & 2 & 2,78 \\
\hline Entre 900 et 1200 & 9 & 12,50 \\
\hline Entre 1200 et 1500 & 6 & 8,33 \\
\hline Entre 1500 et 1800 & 3 & 4,17 \\
\hline Entre 1800 et 2100 & 6 & 8,33 \\
\hline Entre 2100 et 2400 & 3 & 4,17 \\
\hline Entre 2400 et 2700 & 2 & 2,78 \\
\hline Entre 2700 et 3000 & 3 & 4,17 \\
\hline Entre 3000 et 3300 & 0 & 0 \\
\hline Entre 3300 et 3700 & 1 & 1,38 \\
\hline Entre 3700 et 4000 & 2 & 2,78 \\
\hline Entre 3700 et 4000 & 0 & 0 \\
\hline Entre 4000 et 4300 & 0 & 0 \\
\hline Entre 4300 et 4600 & 0 & 0 \\
\hline Entre 4600 et 4900 & 0 & 0 \\
\hline Entre 4900 et 5200 & 2 & 2,78 \\
\hline Total & 72 & 100 \\
\hline
\end{tabular}

Source: Données de l'enquête, août 2017

Il a été difficile pour les caféiculteurs mutualistes de la province Kayanza de nous donner des informations précises sur leurs revenus annuels. Toutefois, les revenus des mutualistes proviennent de la vente des cerises et varient en fonction du nombre de pieds de caféiers, de la qualité de la plantation, cette dernière dépendant à son tour de la qualité du sol et de la capacité de les entretenir. D'autres revenus proviennent de la vente de quelques cultures vivrières.

Lors de notre entretien avec les responsables des cinq mutuelles de santé , nous avons pu comprendre que l'une des facteurs majeurs qui justifie les faibles revenus tirés de la vente des cerises est que lorsque les familles choisissent des parcelles sur lesquelles il faut pratiquer la caféiculture, elles choisissent celles qui sont déjà appauvries par d'autres cultures et situées sur 
des pentes au niveau des collines en réservant d'autres parcelles aux cultures vivrières. Cette réalité fait que les caféiers deviennent très exigeants pour être plus productifs.

Néanmoins, tous nos enquêtés reconnaissent que malgré les exigences en matière de l'entretien de leurs plantations, le café est la seule spéculation qui permet au caféiculteur d'avoir, en une seule saison, une somme d'argent importante qui peut lui permettre de subvenir à ses besoins. Le payement des ouvriers journaliers, la scolarisation des enfants en âge d'aller à l'école, l'achat des denrées alimentaires non produites sur les parcelles familiales, l'achat du matériel pour l'entretien des caféiers, habillement des membres de la famille, la cotisation à la mutuelle, l'achat du petit bétail pour l'élevage, constituent ainsi les principales dépenses réalisées par les mutualistes.

Les revenus de nos enquêtés, leur permettent d'avoir accès à l'eau potable, d'avoir un habitat décent avec de l'électricité pour ceux qui vivent dans des centres urbains. Tous nos enquêtés arrivent à scolariser leurs enfants sauf les enfants en situation de handicape et quelques cas isolés de ceux qui, suite aux faibles revenus des parents abandonnent l'école pour aller travailler comme main-d'œuvre chez les particuliers pour gagner de l'argent.

\section{Identification de circuits de diffusion de l'information au sein des mutuelles de santé}

Tableau 5 : Connaissance de la Fédération des Mutuelles de Santé des Caféiculteurs du Burundi (FEMUSCABU) et identification des circuits d'information

Connaissance de la FEMUSCABU et circuits de diffusion de l'information

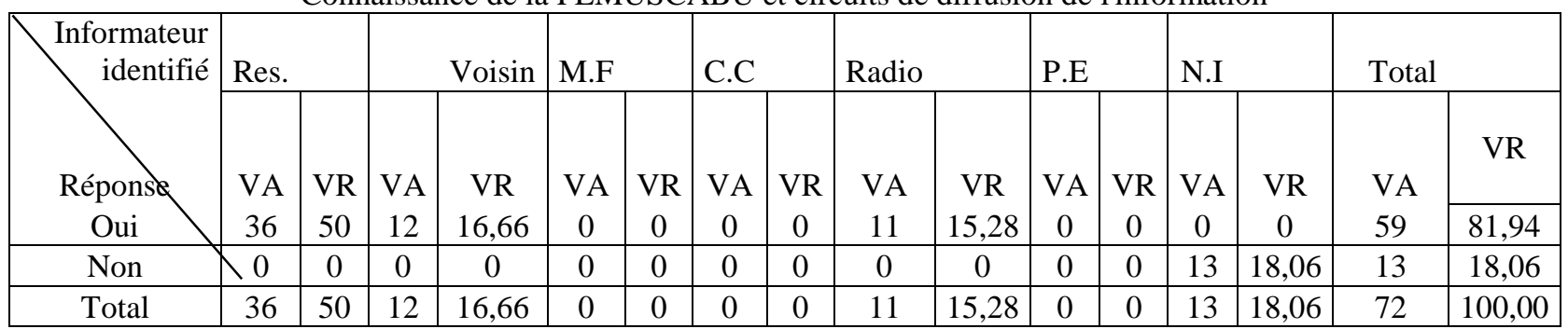

Source: Données de l'enquête, août 2017

Resp. : : Responsable

M.F : Membre de la famille

p.E : Presse écrite

N.I : Non informé

VA : Valeur absolue

VR : Valeur relative (\%)

Source : Données de l'enquête, août 2017

Les données du tableau ci-dessus montrent que sur les 59 enquêtés représentant 81,94\% qui sont informés des activités de la FEMUSCABU, 36 enquêtés ont été informés par les responsables des mutuelles, 12 par les voisins 
et les 11 autres ont été informés par la radio. Par contre, les treize enquêtés représentant $18,06 \%$ affirment ne pas détenir aucune information sur la Fédération des Mutuelles de Santé des Caféiculteurs du Burundi.

Effet, le taux élevé des mutualistes qui ont été informés par les responsables des mutuelles de santé s'explique par le fait que ces responsables ont effectué un travail considérable de sensibilisation des caféiculteurs pour qu'ils adhèrent aux mutuelles de santé. Et comme 65, soit 98,28\% d'enquêtés affirment qu'ils possèdent un poste de radio, les mutuelles de santé devraient diffuser des émissions en rapport avec les avantages que les mutuelles de santé procurent aux mutualistes pour susciter l'adhésion de beaucoup de caféiculteurs aux mutuelles de santé.

En outre, les circuits de communication utilisés au sein des mutuelles de santé sont avantageux dans la mesure où l'information circule horizontalement et verticalement à la fois.

A la question de savoir si les responsables des mutuelles de santé organisent régulièrement des réunions, tous les enquêtés reconnaissent que les réunions s'organisent mais de façon irrégulière. Comme, selon les données de l'enquête, $79,17 \%$ de mutualistes possèdent des téléphones mobiles, les réunions peuvent être convoquées par téléphone pour permettre à tous d'être informé à temps. Cette manière de communiquer va permettre aux $31,95 \%$ qui disent ne pas recevoir des informations via leurs téléphones portables, d'en revoir et être au même niveau d'information que les autres mutualistes.

S'agissant de la question de savoir depuis quand les mutualistes ont adhéré à la mutuelle, les données de l'enquête montrent que la durée d'adhésion varie de une à dix ans. Avec la création de l'Union Grand Nord, les cotisations ont été harmonisées et tous les mutualistes cotisent vingt deux mille cinq cent francs burundais par an.

Tableau 6 : Facteurs explicatifs du non-accès aux soins de santé de qualité

\begin{tabular}{|c|c|c|}
\hline \multirow{2}{*}{ Accès aux soins de santé de qualité } & \multicolumn{2}{|c|}{ Répondants } \\
\cline { 2 - 3 } & Valeur absolue & Valeur relatives (\%) \\
\hline Oui & 63 & 87,50 \\
\hline Non & 9 & 12,50 \\
\hline Total & 72 & 100 \\
\hline
\end{tabular}

Source: Données de l'enquête, août 2017

Les données portées dans le tableau ci-dessus montrent que 87,50\% d'enquêtés reconnaissent avoir accès aux soins de santé dans les centres de santé qui travaillent en partenariat avec leur mutuelles de santé tandis que les $12,50 \%$ d'enquêtés affirment avoir toujours des difficultés à se faire soigner dans les centres de santé partenaires.

Cependant, malgré l'importance que la majorité d'enquêtés reconnaissent aux mutuelles de santé, ils dénoncent tous les maltraitances qu'ils subissent suite au manque d'information de la part des agents de santé 
qui hésitent souvent à considérer leurs cartes mutuelles quand ils sont malades. Cela fait que les mutualistes reçoivent des médicaments insuffisants car leurs cartes sont méprisées par les agents de santé.

En outre, là où les cartes mutuelles leur permettent de se faire soigner c'est-à-dire dans les centres de santé qui ont signé des conventions avec la mutuelle, il n'y a pas de médecins spécialistes alors que certains cas nécessitent l'intervention d'un médecin spécialiste. Aussi, les maladies chroniques ne sont-elles pas prises en charges.

Les caféiculteurs qui ont adhéré aux mutuelles de santé pensaient que le problème d'accès aux soins de santé de qualité étaient fini mais jusqu'aujourd'hui, ils constatent que très peu de centre de santé privé ont accepté de signer la convention avec les mutuelles de santé. Des cas de rupture de stocks de médicaments se produisent souvent, obligeant les mutualistes à aller s'acheter des médicaments dans des pharmacies privés. Certains médicaments comme la quinine par exemple ne sont pas prescrits par les soignants des structures de soins partenaires alors que nos enquêtés, enregistrant des faibles revenus annuels qui ne leur permettent pas d'aller se payer des médicaments dans des pharmacies en cas de rupture de stocks chez les structures de santé partenaires, dénoncent des couts très élevés des médicaments dans les pharmacies. A cela s'ajoute la non prise en charge des maladies chroniques comme le diabète et l'hypertension. Le problème d'insalubrité dans les centres de santé partenaire et l'absence de déontologie professionnelle chez les agents de santé ont été aussi soulignés par nos enquêtés. Soulignons par là que la quinine n'est pas prescrite dans un contexte où le paludisme a été identifié par le Ministère de la Santé et de Lutte Contre le Sida (2012) comme la première cause de mortalité au Burundi.

Nos enquêtés ont aussi souligné leur retard dans le versement de leurs cotisations. Ceci affecte énormément la gestion des mutuelles de santé dans ces périodes où les affiliés ne sont pas encore nombreux.

\section{Contribution des mutuelles de santé à la promotion de l'accès aux soins de santé en province Kayanza}

Les mutuelles de santé des caféiculteurs ont été créées dans l'optique de promouvoir la solidarité et de permettre aux caféiculteurs de se prendre en charge en matière d'accès aux soins de santé de qualité. Le faisant, les mutuelles de santé jouent le rôle d'intermédiaire entre les mutualistes et les structures de soins partenaires. Elles avalisent les mutualistes qui tombent malades lorsqu'ils n'ont de moyens de payer immédiatement les soins. Dans ce souci de permettre aux mutualistes de se faire soigner dans de bonnes conditions, les mutualistes peuvent être soigné à crédit et aller payer après la guérison à condition qu'on garde le carnet jusqu'au jour où l'affilié vient 
payer. Les responsables des mutuelles de santé font le plaidoyer auprès des institutions étatiques et au près des organisations privées.

A travers des réunions, les responsables des mutuelles de santé sensibilisent les mutualistes sur la façon de se protéger contre le paludisme et d'autres maladies. A ces réunions, les responsables des structures de santé partenaires participent et cela leur permet de comprendre la capacité des mutuelles à leur verser leur part. C'est aussi à travers ces réunions que les mutualistes s'informent sur l'état d'avancement des autres mutuelles de santé membres de la FEMUSCABU et d'autres. Etant donné que les centres de santé partenaires travaillent en collaboration avec les pharmacies, les responsables des mutuelles payent à temps ces structures de santé qui doivent se procurer des médicaments au près des pharmacies. Cela à été largement apprécié par nos enquêtés.

$\mathrm{Du}$ point de vu social, les mutuelles de santé interviennent pour appuyer un mutualiste en cas de décès d'un mutualiste ou d'un membre de la famille restreinte en lui achetant par exemple un cercueil.

Comme les 22 autres mutuelles de santé des caféiculteurs, les 5 mutuelles de santé des caféiculteurs membres des différentes associations des caféiculteurs réunis au sein de la fédération Bonakure de la province Kayanza sont, elles aussi, membres de la Fédération des Mutuelles de Santé des Caféiculteurs du Burundi (FEMUSCABU). Cette dernière apporte son appuie aux mutuelles de santé des caféiculteurs de la province Kayanza en finançant et en organisant des campagnes de sensibilisation sur la protection de l'environnement en général et sur la lutte contre le paludisme en particulier.

Comme cela apparait dans un rapport d'une enquête communautaire réalisée par la FEMUSCABU (2013), un des rôles essentiellement assignés à la FEMUSCABU est d'assurer le suivi de la qualité des soins offerts par les formations sanitaires sous-convention avec les MUSCABU.

En cas de manque de moyens d'une mutuelle, la FEMUSCABU appuie matériellement la mutuelle et en collaboration avec l'ADISCO, la FEMUSCABU avance de l'argent aux mutuelles de santé en cas d'incapacité de prendre en charge ses mutualistes. Elle organise des séminaires de formation dans le sens de renforcement des capacités des responsables des mutuelles. Selon les cas, certaines structures de santé qui ont signé une convention de partenariat avec les mutuelles reçoivent des appuis en équipement de la part de la FEMUSCABU. Et c'est dans le souci de permettre aux mutuelles de santé d'atteindre leurs objectifs, qu'une pharmacie des mutuelles à été ouverte à Ngozi. La FEMUSCABU veille donc à la bonne gestion des cotisations des mutualistes. 
Contraintes auxquelles font face les mutuelles de santé des caféiculteurs de la province Kayanza dans leur volonté de permettre à tous les mutualistes d'accéder aux soins de santé de qualité

Lors de notre entretien avec les responsables des mutuelles de santé, ils ont tous été unanimes sur le fait que les effectifs peu élevés des affiliés font que les ressources financiers des mutuelles de santé soient très limitées. A partir des calculs effectués à partir des effectifs des caféiculteurs recensés tel que cela apparait dans le rapport annuel de la Confédération Nationale des Associations des Caféiculteurs (CNAC) de 2013, les caféiculteurs mutualistes ne représentent que $3 \%$ des effectifs totaux des caféiculteurs de la province Kayanza. Dans notre entretien avec le responsable de la mutuelle de santé " Kira' de la commune Gatara, il nous a révélé que le fait que les populations bénéficient de la Carte d'Assurance Maladie (CAM) vendu par les services publics de santé à trois mille (3.000) Francs burundais, la majorité de caféiculteurs n'ont pas encore compris la nécessité d'adhérer à une mutuelle de santé qui exigent une cotisation de 22500 francs burundais par an. Le même interlocuteur a précisé que les mutuelles de santé tirent leurs ressources des cotisations des affiliés, des activités génératrices de revenus, des subventions, des dons et des aides. Alors, si les affiliés sont encore peu nombreux, les cotisations restent insuffisantes pour prendre en charge les mutualistes qui cotisent. L'élan donc de solidarité qui a présidé à la création de ces mutuelles devient difficile à concrétiser.

En plus des CAM de 3000 FBU, il ya aussi la politique du gouvernement burundais qui consiste à prendre en charge les soins de santé de tous les enfants de moins de 5 ans et des femmes enceintes. Ceci constitue une autre raison majeure qui justifie le faible taux d'adhésion des caféiculteurs aux mutuelles de santé. Les CAM qui étaient réservées aux populations à faible revenu, aujourd'hui, même les personnes riches en achètent, disait le responsable de la mutuelle " Kira' de Gatara.

Confrontées en plus aux problèmes de manque de bailleurs, et de rupture de stocks des médicaments dans les structures de santé partenaires, les mutuelles de santé découvrent souvent que ces derniers surfacturent les soins donnés aux mutualistes. En outre, il ya le manque de moyens suffisants et réguliers pour payer les factures qui se combine avec les bas revenus des mutualistes qui ne leur permettent pas de se payer les médicaments dans les pharmacies en cas de rupture de stocks. Les mutuelles de santé éprouvent aussi d'énormes difficultés en cas d'épidémies surtout que jusqu'aujourd'hui, les mutuelles de santé des caféiculteurs n'ont pas encore eu des laboratoires bien équipés qui acceptent de signer une convention avec elles. 


\section{Opinions des mutualistes sur la dynamique de leur mutuelle de santé et leur comportement face aux difficultés d'accès aux soins de santé de qualité}

Tableau 7 : Appréciation de l'assistance que la mutuelle apporte aux mutualistes

\begin{tabular}{|c|c|c|}
\hline \multirow{2}{*}{ Appréciation } & \multicolumn{2}{|c|}{ Répondants } \\
\cline { 2 - 3 } & Valeur absolue & Valeur relatives (\%) \\
\hline Oui & 68 & 94,44 \\
\hline Non & 4 & 5,56 \\
\hline Total & 72 & 100 \\
\hline
\end{tabular}

Source: Données de l'enquête, août 2017

Les données portées dans le tableau ci-dessus, montrent que 94,44\% d'enquêtés apprécient l'apport des mutuelles de santé.

Les $5,56 \%$ de mutualistes qui n'apprécient pas l'assistance que leur apporte leur mutuelle, expliquent que certains médicaments ne sont pas toujours disponibles et que certaines spécialistes comme les ophtalmologues ne sont pas employés au niveau des structures de santé qui ont signé la convention avec les mutuelles.

La non disponibilité des médicaments au niveau des centre de santé pousse les mutualistes à aller les chercher dans les pharmacies et dépensent plus. En outre, les centres de santé qui sont en partenariat avec les mutuelles ne sont pas nombreux et la liberté de choix des mutualistes devient limitée. Mais malgré ces difficultés qui rendent les mutualistes beaucoup plus vulnérables, tous les enquêtés ont affirmé que personne aujourd'hui ne vas se faire soigner chez les charlatans et les féticheurs en province Kayanza. Ce résultat s'oppose à celui obtenu lors d'une enquête menée par Ndabarushimana (2014) dans le cadre de la rédaction de sa thèse unique de doctorat en sociologie.

Face à ces contraintes, les mutualistes souhaitent que les responsables des mutuelles élargissent leur champs d'action en signant des conventions avec d'autres centres de santé et des hôpitaux publics et privés pour que les mutualistes puissent bénéficier des consultations des médecins spécialistes et avoir la facilité dans le choix de centre de santé et de l'hôpital à fréquenter.

En effet, cette demande est justifiée par le fait qu'à l'intérieur du pays, non seulement les moyens financiers sont limités mais aussi et surtout les bus pour assurer les liaisons entre les collines, les zones, et les communes sont quasi inexistants. Les quelques taxis voiture appelés communément "kagongo" et les taxis motos qui assurent ces liaisons restent rares et par conséquent sont chers.

Pour avoir accès aux soins de santé de qualité, les mutualistes suggèrent que la FEMUSCABU assistent les mutuelles de santé dans la mise en place de leurs propres pharmacies pour que leur couverture puisse s'étendre 
jusqu'à l'achat des médicaments qui ne sont pas disponibles dans les centres de santé partenaires.

Les 94,44\% d'enquêtés qui apprécient l'assistance leur apportée par leur mutuelles justifient que les responsables paient les factures à temps pour que les mutualistes continuent d'être soignés par les structures de santé partenaires. Le fait que les responsables des mutuelles organisent des campagnes de sensibilisation sur la manière dont il faut prévenir les maladies, cela permet aux mutualistes de prendre soins d'eux-mêmes et de se protéger contre par exemple les piqûres des moustiques.

A la question de savoir ce que les mutualistes reprochent aux responsables des mutuelles, les réponses données par les enquêtés ont été codées en deux mots, à savoir : l'irrégularité et la descente sur le terrain.

Les irrégularités dénoncées par quelques mutualistes s'observent, d'une part, lorsque les responsables ne sont pas au bureau lorsque les mutualistes ont besoin d'eux ; et d'autre part, lorsqu'ils tardent à convoquer des réunions ordinaires. Les réunions des mutualistes s'organisent semestriellement mais il ya des fois où les retards s'observent selon quelques mutualistes.

Selon les mutualistes, les responsables des mutuelles de santé descendent rarement sur le terrain pour rencontrer et écouter les doléances des mutualistes. Cela justifierait aussi le fait qu'il y ait des caféiculteurs qui n'adhèrent pas aux mutuelles de santé.

Selon le rapport annuel de la Confédération Nationale des Associations des Caféiculteurs du Burundi (2015), la fédération des associations des caféiculteurs « BONAKURE » de Kayanza qui couvre notre champ d'étude est composée de 27 unions de caféiculteurs réunis au sein de 677 associations. Toutes ces structures rassemblent actuellement 21347 caféiculteurs. Etant donné que dans la base des données de l'ADISCO et de l'Union Grand Nord qui sont des structures d'encadrement des mutuelles de santé, étaient enregistrés, au cours de l'Exercice 2017, seulement 717 caféiculteurs mutualistes, il ya lieu de chercher à savoir les facteurs justificatifs de ce faible taux d'adhésion des caféiculteurs aux mutuelles de santé. Partant donc des effectifs ci-haut mentionnés, le taux d'adhésion des caféiculteurs aux mutuelles de santé en 2016 était de 3\%.

\section{Discussion des résultats}

L'analyse de la dynamique des mutuelles de santé des caféiculteurs de la province Kayanza met en relief les caféiculteurs mutualistes issus des 27 unions des associations des caféiculteurs de la province Kayanza et les responsables des mutuelles de santé qui ont pris l'initiative de les mettre en place depuis 2008. Ces unions sont réunies au sein de la fédération Bunakure de la province Kayanza. Comme il s'agit des mutuelles des caféiculteurs, tous 
les mutualistes sont en même temps membres des associations des caféiculteurs.

En effet, dans la société burundaise, le café a toujours été considéré comme étant une culture de rente c'est-à-dire une culture qui génère assez de revenus groupés pour les familles, pouvant leur permettre ainsi de s'acquitter de leur cotisation et de subvenir à leurs besoins fondamentaux. Ces résultats coïncident avec ceux de Kimonyo \& Ntiranyibagira (2007) qui ont souligné que "Pour le caféiculteur, le café a constitué pendant longtemps la principale source de revenus familiaux surtout dans les régions du nord du pays, en particulier à l'époque où les produits vivriers étaient abondants et donc peu chers sur le marché (...). L'intérêt de la culture du café pour le paysan burundais réside encore sur le fait que la récolte caféière est saisonnière et fournit des recettes groupées que le caféiculteur n'est pas en mesure d'épargner tout au long de l'année'.' (p. 8).

Et comme la société burundaise est une société patriarcale, tout ce secteur est contrôlé par les hommes, d'où les hommes qui adhèrent aux mutuelles de santé restent majoritaires. Le payement des ouvriers journaliers, scolarisation des enfants en âge d'aller à l'école, achat des denrées alimentaire non produites sur les parcelles familiales, achat du matériel pour l'entretien des caféiers, habillement des membres de la famille, cotisation de la mutuelle, achat du petit et du gros bétail pour l'élevage, constituent les principaux dépenses réalisées par les mutualistes.

Le fait que le taux d'adhésion aux mutuelles de santé jusqu'en 2017 n'était que de $3 \%$, cela affectent énormément la gestion des mutuelles de santé qui se retrouvent souvent sans moyens financiers pour payer les factures émises par les centres de santé partenaires. Les cotisations des mutualistes sont irrégulières alors que le fondement même d'une mutuelle de santé est cette prise en charge mutuelle qui permet aux responsables des mutuelles de régler les factures à base des cotisations déjà collectées.

Parmi les missions des responsables des mutuelles de santé des caféiculteurs figurent le plaidoyer et la diffusion de l'information relative aux mutuelles de santé. Nous avons trouvé que dans le cadre de la gestion des mutuelles de santé, l'information circule aussi dans les deux sens, vertical et horizontal. L'article d'Ibrahima, Oumar, Mamadou, \& Anta Tal (2017) nous renseignait déjà sur le fait que le manque d'information avait été identifié comme étant le premier motif de non adhésion aux mutuelles de santé dans la région de Ziguinchor au Sénégal.

Aussi, de ombreuses études faites en Afrique de l'Ouest et au Ghana, disait-il, ont-elles démontré que la faible compréhension et connaissance des concepts et principes qui sous-tendent les dispositifs d'assurance maladie sont la principale raison de la faible adhésion aux mutuelles de santé. Pour cela, nous estimons que pour ne pas rester dans les mêmes difficultés que les 
populations de Ziguinchor, les responsables des mutuelles de santé, appuyés par l'administration locale, doivent mettre l'accent sur les canaux de communication les plus accessibles et les plus utilisés par la population notamment les radios communautaires et les téléphones mobiles pour mobiliser à outrance les caféiculteurs qui n'ont pas encore adhéré aux mutuelles de santé pour qu'ils rejoignent les autres caféiculteurs dans cet élan de solidarité et d'autodétermination communautaire. L'usage des radios communautaires comme outils de communication a permis aux mutuelles de santé de connaître une expansion sans précédent au cours de ces dernières décennies comme nous le révèle encore une fois Ibrahima et al. (2017).

Les résultats obtenus dans cet article montrent que 94,44\% d'enquêtés apprécient l'assistance leur apportée par leur mutuelles. Ces résultats concordent avec ceux de Awomo Ndongo, Mahieu, \& Nanfosso, 2014). Les résultats obtenus par ces auteurs ont prouvé que l'adhésion à une mutuelle de santé a un effet positif sur l'état de santé de l'individu. Ainsi, le fait d'adhérer à une Mutuelle de Santé augmente d'environ 9\% la probabilité que l'état de santé de l'individu soit meilleur par rapport à celui qui n'adhère pas chez les populations du centre du Cameroun. Ceci peut se justifier par un meilleur accès aux soins, mais également par une meilleure qualité des soins qui est offerte dans les formations sanitaires conventionnées c'est-à-dire sous contrat avec les mutuelles de santé. Cependant, ces auteurs ont révélé que ces résultats étaient aussi similaires avec ceux obtenus par d'autres chercheurs en Inde et aux Philippines. Par contre, ces résultats, selon les mêmes auteurs, ne coïncident pas avec ceux trouvés au Kenya, au Rwanda et au Cambodge.

Dans notre thèse unique de doctorat, nous avions déjà montré que l'irrégularité observée dans le payement des caféiculteurs les rend tellement vulnérable de telle sorte qu'ils pouvaient vendre aux plus nantis leur ticket d'une valeur par exemple de cinquante mille francs burundais à un prix dérisoire avec une perte de près d'un tiers de la valeur totale du ticket (Ndabarushimana, 2014). Les revenus du caféiculteur donc depuis la présentation de ma thèse en 2014 , ne sont pas encore maîtrisés et ne permettent pas au caféiculteur d'adhérer à une mutuelle de santé qui exige une cotisation annuelle de 22500 francs burundais alors que la Carte d'Assurance Maladie donnée par l'Etat lui coûte 3000 francs burundais. Cette situation d'insuffisance et d'irrégularité des revenus du caféiculteur mutualiste le pousse à se contenter des avantages que le gouvernement burundais accorde à toute la population en matière d'accès aux soins de santé. Il s'agit notamment de la gratuité des soins de tous les enfants de moins de cinq ans et de la gratuité des soins des femmes enceintes jusqu'à l'accouchement.

En plus de l'absence des médecins spécialistes, dans les conventions signées entre les mutuelles de santé et les centres de santé, les maladies chroniques ne sont pas prises en charge et cela fait penser aux mutualistes que 
les mutuelles de santé ne sont pas encore capables de répondre efficacement à leurs besoins en matière d'accès aux soins de santé.

A cet égard, la publication de Handicap International (2016) est une référence fort pertinente sur la souffrance qu'endurent jusqu'aujourd'hui les femmes et les enfants. Selon cette organisation, les femmes et les enfants de moins de 5 ans paient un lourd tribut. Les maladies à évolution chronique telles que le sida, la tuberculose, le diabète, l'hypertension artérielle, l'asthme, les cardiopathies, les maladies mentales, ainsi que les violences physiques sexuelles, accidents sur la voie publique, guerre - sont à l'origine de nombreuses incapacités physiques et mentales. Il est vrai que l'adhésion des caféiculteurs de la province Kayanza aux mutuelles de santé n'a pas résolu tous les problèmes d'accès aux soins de santé, mais a permis aux caféiculteurs ayant de faibles revenus, de pouvoir se faire soigner sous l'effet combiné des efforts des autres mutualistes qui cotisent régulièrement dans leurs mutuelles de santé.

Les résultats atteints par l'Appui au Développement Intégral et à la Solidarité sur les Collines (2013) rejoignent ceux obtenus dans cet article.

En effet, sur les cinq variables d'analyse retenues, la province Kayanza occupait la première place sur la première, deuxième, troisième, cinquième et sixième variable. Elle occupe la quatrième place pour la quatrième variable sur les cinq champs concerné par l'enquête.

Les résultats de cet article montrent que le niveau d'étude est une variable déterminant dans l'adhésion à une mutuelle de santé. En outre, parmi les caféiculteurs de la province Kayanza ayant adhéré aux mutuelles de santé, 57 , soit $79,16 \%$ ont 4 enfants et plus.

En effet, cette réalité sociale est similaire à celle qui prévaut dans la région de Ziguinchor au Sénégal où Ibrahima et al. (2017) ont trouvé que les ménages avec un niveau d'instruction et de revenus élevés, d'une taille inférieure à 10 personnes, un engagement social important et une fréquence élevée de consultation pour les problèmes de santé étaient plus à même d'adhérer à une mutuelle de santé.

Parmi les missions des responsables des mutuelles de santé des caféiculteurs figurent le plaidoyer et la diffusion de l'information relative aux mutuelles de santé. Nous avons trouvé que dans le cadre de la gestion des mutuelles de santé, l'information circule aussi dans les deux sens, vertical et horizontal. Toutefois, les ruptures répétitives des stocks de médicaments dans un contexte où les structures de soins partenaires restent peu nombreuses rendent davantage les mutualistes vulnérables.

\section{Conclusion}

La présente étude a contribué à une meilleure connaissance de la dynamique des mutuelles de santé des caféiculteurs de la province Kayanza. 
Elle a fourni des informations utiles aux responsables des mutuelles de santé, de la Fédération des Mutuelles de Santé des Caféiculteurs du Burundi ainsi qu'aux décideurs politiques qui ont le devoir de veiller à ce que toutes les couches sociales ont accès aux soins de santé de qualité.

Dans un sens historique, notre étude a montré comment les caféiculteurs de la province Kayanza se sont organisés progressivement de la base au sommet, d'abord en tant que caféiculteurs, ensuite en tant que mutualistes pour répondre aux problèmes liés à l'accès aux soins de santé de qualité. Les revenus des caféiculteurs qui restent bas, l'irrégularité des cotisations, le taux d'adhésion des caféiculteurs aux mutuelles de santé reste insignifiant, le mépris de certains personnel des structures de santé à l'égard des cartes mutuelles des caféiculteurs, les maltraitances qu'ils subissent suite au manque d'information de la part des agents de santé qui refusent de considérer leurs cartes mutuelles, le manque de médecins spécialistes, les ruptures de stocks de médicaments, le fait qu'il y ait très peu se structures de santé qui acceptent de signer la convention avec les mutuelles de santé, les couts très élevés des médicaments dans les pharmacies, la non prise en charge des maladies chroniques, sont autant de facteurs identifiés qui empêchent les caféiculteurs mutualistes de la province Kayanza d'avoir accès aux soins de santé de qualité.

Les mutuelles de santé des caféiculteurs ont été créées dans l'optique de promouvoir la solidarité et de permettre aux caféiculteurs de se prendre en charge en matière d'accès aux soins de santé. Le faisant, les mutuelles de santé jouent le rôle d'intermédiaire entre les mutualistes et les centres de santé partenaires. Cette étude a montré également que les mutualistes peuvent être soigné à crédit et aller payer après à condition qu'on garde le carnet jusqu'au jour où l'affilié vient payer. Les responsables des mutuelles de santé font le plaidoyer auprès des institutions étatiques et au près des organisations privées. D'ailleurs, un des rôles essentiellement assignés à la FEMUSCABU est d'assurer le suivi de la qualité des soins offerts par les formations sanitaires sous-convention avec les MUSCABU.

Notre étude a également élucidé un certains nombre de contraintes auxquelles font face les mutuelles de santé dans leur mission de promouvoir l'accès aux soins de santé des caféiculteurs mutualistes en province Kayanza. C'est notamment les ressources financières qui restent insuffisant suite aux mutualistes qui sont encore peu nombreux mais aussi et surtout, à cause des irrégularités des cotisations des mutualistes. Aujourd'hui, de nombreux caféiculteurs ne sentent pas la nécessité d'adhérer aux mutuelles de santé des caféiculteurs à cause de la Carte d'Assurance Maladie qui coûte moins cher par rapport au montant exigé par les mutuelles comme cotisation annuelles. Les mutuelles de santé éprouvent aussi d'énormes difficultés en cas d'épidémies. 
Comme les structures de santé partenaires des mutuelles de santé ne sont pas encore nombreuses à Kayanza, les mutualistes n'ont pas de la liberté de choix et la rupture de stocks de médicaments oblige les mutualistes à aller chercher les médicaments dans les pharmacies privées et dépensent plus.

Face à ces contraintes, les mutualistes souhaitent que les responsables des mutuelles élargissent leur champs d'action en signant des conventions avec d'autres structures de santé et des hôpitaux publics et privés pour que les mutualistes puissent bénéficier des consultations des médecins spécialistes et avoir la facilité dans le choix de structures de santé à fréquenter.

Pour avoir accès aux soins de santé de qualité, les mutualistes suggèrent que la FEMUSCABU assistent les mutuelles de santé dans la mise en place de leurs propres pharmacies pour que leur couverture puisse s'étendre jusqu'à l'achat des médicaments qui ne sont pas disponibles dans les centres de santé partenaires.

Pour faire face à cette série d'obstacles qui empêchent le caféiculteur mutualiste de la province Kayanza d'avoir accès aux soins de santé de qualité, les décideurs politiques, en collaboration avec les responsables des structures ayant hérité de la gestion du secteur café au Burundi depuis la promulgation de la loi $\mathrm{n}^{\circ} 100 / 012$ du 14 janvier 2005 portant réforme de la filière café, doivent envisager la mise en place d'une stratégie de communication adaptée pour amener les caféiculteurs à adhérer aux mutuelles de santé qui sont les leurs. Plus ils seront nombreux à adhérer, plus les ressources vont augmenter à travers les cotisations et permettront par conséquent aux mutualistes d'avoir accès aux soins de santé de qualité et feront entendre leur voix en cas de plaidoyer. De même, les caféiculteurs devraient prendre conscience que seuls leurs efforts pourront servir de pierres angulaires pour avoir des partenaires qui comprennent leur cause et qui sont capables de soutenir leurs efforts, car comme le disait Mendras (1992), “le bon paysan est courageux" (p. 243).

\section{References:}

1. Arnaud, Z., \& Rigot, V. (2007). La privatisation de la filière café au Burundi. Bruxelles: Centre National de Coopération au Développement.

2. Assogba, Y. (2008). Développement communautaire en Afrique. Comprendre la dynamique des populations. Québec : Les Presses de l'Université Laval.

3. Awomo, N.J-.C., Mahieu, P.-A., \& Tsafack, N. R. (2014). Mutuelles de santé et état de santé des populations au Cameroun : une enquête conduite dans la région du Centre pour estimer l'effet de l'adhésion à une mutuelle sur l'état de santé déclaré, in, Journal de gestion et d'économie médicale, 2014/4 (Vol. 32). Yaoundé : Université de Yaoundé II, REMA. 
4. Bachelard, G. (1980). La formation de l'esprit scientifique. (10 ème éd.). Paris : Librairie philosophique J. VRIN.

5. Beaud, S. \& Webert, F. (2003). Guide de l'enquête de terrain. Paris : La Découverte.

6. BIT, (2002). Micro-assurance santé. Guide d'introduction aux mutuelles de santé en Afrique. Programme Stratégies et Techniques contre l'Exclusion sociale et la Pauvreté (STEP). Genève : BIT.

7. CNAC_Murima w'isangi, (2015). Rapport annuel.

8. Constitution du Burundi promulguée le 18 mars 2005.

9. Cochet, H. (2001). Crises et révolutions agricoles au Burundi. Paris: Karthala.

10. Defourny, J. \& Failon, J. (2011). Les déterminants de l'adhésion aux mutuelles de santé en Afrique subsaharienne: un inventaire des travaux empiriques. In Mondes en développement 2011/1 ( $\left.n^{\circ} 153\right)$.

11. Ibrahima, S. (2017). Déterminants de l'adhésion et de la fidélisation aux mutuelles de santé dans la région de Ziguinchor (Sénégal), in Santé publique 2017/1 (Vol. 29). S.F.S.P.

12. Javeau, C. (1990). L'enquête par questionnaire. Manuel à l'Usage du Praticien. $4^{\text {ème }}$ éd. Bruxelles : Editions de l'Université de Bruxelles.

13. Kaneza, E. (2013). Les unions des mutuelles de santé des caféiculteurs dans le suivi de la qualité des soins, in la voix des collines, trimestriel d'information, de formation et d'action $d u$ monde rural $n^{\circ} 8$, juillet août - septembre. Bujumbura.

14. Ministère de l'Agriculture et de l'Elevage (2011). Plan National d'Investissement Agricole (PNIA) 2012 - 2017. Bujumbura.

15. Karera, D. \& Al. (2014). Encourager l'intensification de la production du café et des cultures vivrières par l'utilisation de la fumure organique dans les zones couvertes par le projet cfc/oic/30 au Burundi. Rapport final du projet au Burundi. Période couverte: Juillet 2012 à Décembre 2013. Bujumbura.

16. Mendras, H. (1992). La fin des paysans. Saint-Amand-Montrond : Babel.

17. Mondjanagni, A.-C. (1984). La participation populaire au développement en Afrique noire, in, Cahiers de l'Institut Panafricain pour le Développement (IPD) ; 23 - 49. Paris: Karthala.

18. Mucchielli, A. (1991). Les méthodes qualitatives. Paris : PUF.

19. N'da, P. (2002). Méthodologie de la recherche. De la problématique à la discussion des résultats. Comment réaliser un mémoire, une thèse en Sciences sociales et en Education. Abidjan : EDUCI.

20. Ndabarushimana, A. (1999). Programme d'Ajustement Structurel et

21. Appauvrissement au Burundi. Travail de Fin de Cycle (TFC). Nairobi :

22. Université Internationale Espoir. 
23. Ndabarushimana, A. (2014). Innovations techniques caféicoles et développement rural: Etude du cas de l'expérience burundaise à travers l'exemple de la fédération bonakure de la province kayanza au nord $d u$ Burundi. Thèse unique de doctorat en sociologie, option, sociologie rurale. Université Félix Houphouët Boigny d'Abidjan/Cocody. Abidjan. P278.

24. Ouellet, A. (1987). Processus de recherche une approche systématique. Québec : Presses Universitaires de Québec.

25. REPUBLIQUE DU BURUNDI, CSLP I, (2007).

26. REPUBLIQUE DU BURUNDI, CSLP II, (2012).

27. Sobngwi, T, P. (2011). Les mutuelles de santé promues par le SAILD dans la région de l'ouest au Cameroun : viabilité et perceptions par les populations. Mémoire d'Ingénieur Agronome, option: Economie et Sociologie Rurales. Yaoundé. Université de Dschang. 